\title{
Saccadic Eye Movements of Children and Adults to Double-Step Stimuli
}

\author{
Susan L. Groll and Leonard E. Ross \\ University of Wisconsin-Madison
}

\begin{abstract}
The programming and reprogramming of oculomotor responses to double-step and single-step targets was investigated in 5-6-year-old and 10-12-year-old children and in adults. The independent variables in Experiment 1 were intertarget interval $(50,100,150$, and $200 \mathrm{msec})$ and target location. The number of trials on which a saccade was made to both first and second targets increased with age and intertarget interval, but the two factors did not interact. On trials where responses were made only to the second target, the children responded slower than the adults but showed generally similar patterns of response latencies. In Experiment 2, a warning signal was presented 0,100 , or $300 \mathrm{msec}$ prior to the first target. For adults the 100- and 300-msec warning intervals reduced the latency of single-step responses and the first saccade of double-step responses, whereas only the 300 -msec warning interval was similarly effective with children. In both experiments subjects in all age groups exhibited amplitude transition functions, indicating that the modifiability of saccadic programming is basically similar for adults and children. A comparison of simultaneous programming characteristics of adults and children suggested possible age differences, but the data were not conclusive. The results indicated age differences in the rate of programming and reprogramming saccades but no qualitative age-related differences in these processes.
\end{abstract}

Although there is convincing evidence that the complex patterns of eye movements involved in visual scanning show developmental changes (e.g., see Day, 1975; Vurpillot, 1968), there has been little investigation of possible concomitant developmental changes in the programming mechanisms that determine the direction and amplitude of individual saccades or in the modifiability of such programming upon the receipt of new target information. Some differences in the saccadic systems of infants and adults have been demonstrated (e.g., Aslin \& Salapatek, 1975), but the characteristics of saccades and the factors related to their programming have been investigated in only a

This research was supported by U. S. Public Health Service Grant No. HD0240 to the second author and is based on a thesis submitted by the first author in partial fulfillment of the requirements for a Master's degree at the University of Wisconsin-Madison.

The authors express their appreciation to Susan Ross for her assistance in carrying out the research.

Requests for reprints should be sent to Susan Groll, who is now at the Learning Disorders Unit, Department of Pediatrics, Yale University School of Medicine, 333 Cedar Street, New Haven, Connecticut 06510. cursory manner in older children and then primarily in terms of such factors as the latency of saccades to peripheral targets under various stimulus conditions (e.g., Cohen \& Ross, 1977; Miller, 1969).

It is clear that visual scanning is a complicated behavior that involves a variety of cognitive and visual processes that interact in a complex manner. Visual scanning contributes importantly to cognitive activity in bringing the relevant visual material into foveal regard and permitting the necessary intake and comparison of visual information. In addition it has been suggested that the eye movements involved in scanning play an important role in the development of schemas (Jeffrey, 1968) and that eye-movement motor activity is a correlate of perceptual development (see Whiteside, 1974, for a brief review of such theories). Thus interest in children's eye movements typically has been directed toward more complex scanning behaviors as they reflect developmental changes in the functional aspects of eye movements and the attentional and cognitive factors that affect them. 
Although developmental differences in scanning may primarily reflect changes in higher level cognitive functioning of children, it is also possible that specific characteristics of the eye-movement control system change with age and experience and play an important role in more complex visual activities such as scanning. If this is the case, the study of eye movement programming might be of value in helping to understand the processes underlying the more complex developmental scanning differences. In addition, identification of the characteristics of the saccadic programming processes of nonhandicapped children could prove useful in identifying problems of handicapped children who may suffer various visual control difficulties.

The present study's purpose was the investigation of aspects of children's and adult's saccadic programming, especially with respect to characteristics of the reprogramming process. One important aspect of the planning and execution of saccades is the manner in which the ongoing programming of eye movements. can be modified by the occurrence of new target information. Whereas early views of the saccade stressed its ballistic nature (i.e., that the system was refractory to changes in target information during the programming and execution of a saccade), more recent studies (e.g., Becker \& Fuchs, 1969; Becker \& Jürgens, 1979; Carlow, Dell-Osso, Troose, Daroff, \& Birkett, 1975; Komoda, Festinger, Phillips, Duckman, \& Young, 1973; Taumer, 1975; Wheeless, Boynton, \& Cohen, 1966) have demonstrated that ongoing saccadic programming can be modified and that the parallel programming of saccades can occur under some circumstances.

The demonstration of the modifiability of the saccadic programming of adults to a large extent has utilized the double-step paradigm in which two targets are successively presented as horizontal displacements from an initial fixation point. Prior work with adults using the double-step paradigm found that the interval between the two targets influenced the probability that a saccade would be made to both targets. At longer intertarget intervals, saccades are made to both the first and second targets; but as this interval decreases the probability of a single saccade to the final target location increases (Carlow et al., 1975; Komoda et al., 1973; Wheeless et al., 1966). The occurrence of single responses to the final target location has been viewed as an indication that the saccadic system processes new information in a more or less continuous manner, and comparisons of the latency of such reprogrammed responses to second targets when these targets appear at various locations have been used to estimate the time needed to cancel and reprogram a saccade. It has been shown that when reprogramming involves recomputing the direction of the saccade (i.e., when first and second targets occur in different retinal hemifields), response latency is greater than that of responses that do not involve reprogramming (Carlow et al., 1975; Komoda et al., 1973; Wheeless et al., 1966). On the other hand when reprogramming involves an extent change but no direction recomputation, the saccade is executed in less than normal programming time (Komoda et al., 1973).

In addition to these findings, a recent study by Becker and Jürgens (1979) has presented data indicating that the amplitude of the saccadic response is related to the delay (D) between the second target step and the onset of the first response. Thus for short intervals the amplitude of the saccade approximates the first target position; but as $D$ lengthens a gradual change in amplitude, called an amplitude transition function, is observed with amplitude increasing or decreasing until it finally matches the second target position. This continuous amplitude change, which was interpreted as demonstrating that the saccadic system continuously processes visual information, was found when the first and second targets occurred in the same retinal hemifield but not when they occurred in different retinal hemifields. A second important relationship reported by Becker and Jürgens was a decrease in the intersaccadic interval between the first and second double-step saccades as D increased. Because parallel processing of the first and second responses could only take place during the $D$ interval, the finding of a decrease in the intersaccadic interval with increasing $D$ was taken as evidence for the simultaneous programming of the saccades.

The present experiments were conducted 
to determine if there were age differences in the saccadic responses to double-step stimulation that would suggest developmental differences in saccadic programming and reprogramming mechanisms. In Experiment 1 children's and adults' saccadic responses to various single- and double-step target sequences were examined. Experiment 2 investigated the effects of warning signals on the saccadic responses of children and adults under similar single- and double-step conditions, since it has been demonstrated that the latencies of children's saccades to single targets can be shortened to adult values if a warning signal preceeds the target (Cohen \& Ross, 1977, 1978).

\section{Experiment 1}

\section{Method}

\section{Subjects}

Subjects consisted of one group of five adults (two males, three females) who were recruited from the university student population and two groups of children who were recruited through signs placed in apartment buildings. The younger group of children, ages 5-6 years, consisted of two boys and three girls $(M$ age $=$ 5.8 years, $S D=.36$ ); and the 8-10-year-old group consisted of three boys and four girls $(M$ age $=9.2$ years, $S D=1.03$ ). Each subject was paid $\$ 2$ or, in the case of children who so chose, given a toy. All subjects were required to have at least $20 / 30$ vision, without corrective lenses, as measured by a Titmus tester.

\section{Apparatus}

Subjects sat in an ophthalmologist's examination chair, which was located $92 \mathrm{~cm}$ from the screen of a Hewlett Packard Display Oscilloscope. The oscilloscope, which was used to present the stimuli, contained a fast decay (to $10 \%$ of original intensity in $2.8 \mathrm{msec}$ ) P15 phosphor screen. Black cardboard covered all of the screen except for a $35 \times 2 \frac{1}{2} \mathrm{~cm}$ rectangular area located at the subject's eye level. The experimental room was dark except for a 15-watt lightbulb, which was not in the subject's sight.

The stimulus-generating and response-recording equipment was located in an adjacent control room, with an intercom permitting communication between the experimenter and the subject. The stimuli presented on the display screen were generated and timed by a laboratory computer.

The subject's eye movements were detected using a Narco Biometric Eye Trac Model 200 eye-movement monitor. Horizontal eye movements were recorded from the right eye, and vertical eye movements were recorded from the left eye. During each trial, the unfiltered Eye
Trac output for the right eye (horizontal eye movements) was sampled every $5 \mathrm{msec}$ and digitized by the laboratory computer, which identified the onset of the first saccade and determined its latency. At the conclusion of the trial, the trial conditions and saccade latency were printed, and the saccade, with the computer-determined saccade onset identified, was displayed on a monitor scope for the experimenter's inspection. Permanent records of the unfiltered analog output of the Eye Trac were made using a Beckman R511A dynograph. The paper was run at $50 \mathrm{~mm} / \mathrm{sec}$, which permitted latency measurement with an accuracy of \pm 10 msec. The polygraph record was used to score responses whenever the computer printout and/or monitor display was ambiguous.

\section{Stimuli}

A dot at the center of the display screen served as the fixation point. At the onset of a trial, the fixation point was extinguished and immediately reappeared as the first target at another position on the screen. The new target position was always a horizontal displacement of the fixation point. On double-step trials, the target then moved to a second horizontal displacement; and the fixation point immediately reappeared at the final target offset.

The two types of stimulus displacement were doublestep and single-step. For double-step trials the first target appeared for a duration of $50,100,150$, or $200 \mathrm{msec}$, following which time the second target appeared for 1 $\mathrm{sec}$. There were two types of double-step stimuli. In one, pulse-over-return, the first target consisted of a $6^{\circ}$ horizontal displacement, followed by a $12^{\circ}$ displacement in the opposite direction. In the other, the pulse-partialreturn, the initial $6^{\circ}$ displacement of the target was followed by a $3^{\circ}$ displacement in the opposite direction. The initial direction of movement was equiprobable. For single-step trials there was only one target, which consisted of a $3^{\circ}$ or $6^{\circ}$ displacement in either direction. These stimulus patterns, and representative responses from one subject, are presented in Figure 1; which also indicates the notation that will be employed for the various response types. Subjects viewed a present sequence of 120 stimuli consisting of 40 single-step trials and 80 double-step trials, with equal numbers of each intertarget interval and target direction. The stimuli were arranged in a Fellows (1967) sequence in order to prevent anticipation of initial direction of movement or stimulus type.

\section{Procedure}

All subjects completed the trials in one session, which began with the experimenter obtaining the subject's or, in the case of children, a parent's written consent. The administration of a visual acuity test followed, with only those subjects with $20 / 30$ or better vision continued in the study. Subjects were then seated facing the display oscilloscope and instructed to fixate the central dot (fixation point) and to follow it quickly whenever and wherever it moved without moving their heads. Children were given practice tracking the experimenter's finger to be certain that they understood the instructions. 


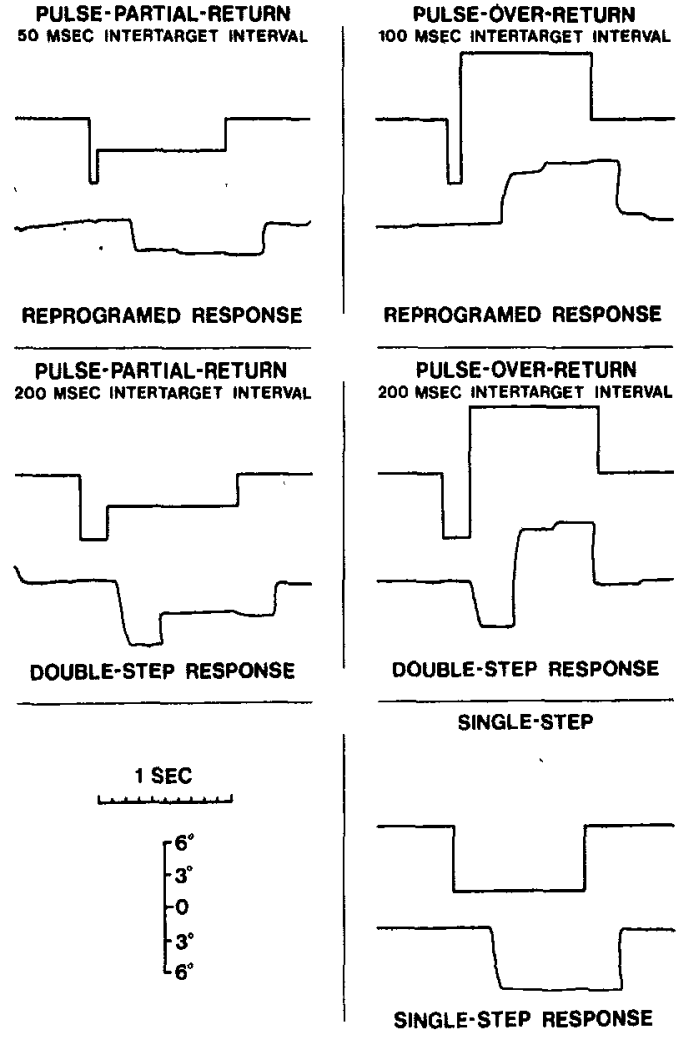

Figure 1. Single- and double-step stimulus patterns and representative responses from one subject. (On pulsepartial-return or pulse-over-return trials [both are double-step trials] there may be saccades to both targets [double-step-responses] or the response to the first target may be canceled and a single response made to the second target [a reprogrammed response]).

The eye-movement sensors were adjusted using a calibration array of the numbers $1-5$. The numbers were equally spaced and arranged horizontally with the 3 in the center of the screen and 1 and 5 located at opposite ends of the screen, each $5^{\circ}$ from the 3 . Once the equipment was adjusted, subjects were informed that the experiment was about to begin. Each trial started with a computer check of the subject's eye position. If it was more than $.75^{\circ}$ from the fixation point, the computer did not present the stimuli. Instead, the experimenter waited for the subject to refixate, following which time the trial was initiated. If the subject's gaze still was not within the fixation area, trials were halted briefly; and if necessary the Eye Trac sensors were recentered to compensate for changes in the subject's head position. Children were given a 15-30 minute break after 60 trials. The intertrial interval was approximately $10 \mathrm{sec}$ and the entire procedure, not including the child's break, took place in 20-30 minutes.

Previous work in this laboratory has determined that children fatigue quickly when a chin rest or bite bar is used to maintain head position. The required posture is uncomfortable for young children and reduces the time they will fully cooperate in the task. Thus a head rest that firmly positioned the head between two cushions was used in the present study. Children evidenced no difficulty in maintaining head position under these conditions, a fact that was verified both by the computer check of eye position at the onset of each trial and by examination of eye movement records during the experimental trials. Because the apparatus employed measured eye position relative to head position, any movement of the head changed the relationship between calibrated eye position and target position; and the computer responded as if the subject was no longer looking at the fixation point. Head movements were then indicated by a sequence of attempted trials in which the computer did not present the stimuli because eye position was incorrectly determined to be outside the area of the fixation point. In addition, head movements during a saccade could be identified from the polygraph records, since in these studies as in previous work, saccades associated with head movements were found to produce a record discriminately different from those without such movement. The few trials with records that indicated head movements were not included in the analyses.

\section{Results and Discussion}

The percentage of double-step responses at each intertarget interval was computed, saccadic latencies were either scored by computer or measured from the polygraph records, and saccadic amplitude was measured from the polygraph records. Any trial that was ambiguous either in terms of response type or latency was discarded; and the data of one adult, with more than 10 discarded responses, was replaced. Preliminary analysis of left-right data revealed no significant differences, and all response measures were averaged over initial direction of stimulus displacement.

\section{Percentage of Double-Step Responses}

The percentage of double-step responses to successive target displacement has been found to be related to the interval between the first and second targets. Presumably at shorter intertarget intervals, the response to the first target is likely to be canceled and reprogrammed, which results in the execution of a single response made to the second target. As the interval increases and more time is available for its programming, the first saccade of the double-step response is increasingly likely to occur. It is of interest to examine the percentage of double-step 
responses made by children and adults since the saccadic latency of children is longer than that of adults, and it might be expected that their programming of the response to the first target would be less well developed at the onset to the second target. A reprogrammed response to the second target thus would be more likely, and the percentage of double-step responses would be less for children at each intertarget interval.

Because an analysis of variance (ANOVA) conducted on the double-step data revealed no effect of stimulus type for any of the three groups (all $p s>.10$ ), the percentages of double-step responses were averaged over that factor. The resulting functions, relating the probability of double-step responding to the interval between the first and second targets, are presented in Figure 2. As can be seen, each of the three age groups showed an increase in percentage of double-step responses as intertarget interval increased from 50 to $200 \mathrm{msec}$, with the level of double-step responding consistently higher for older subjects. Multiple regression/correlation analysis of these data, with age as a between factor and intertarget interval as a within factor, revealed a significant main effect of both intertarget interval, $F(3,42)=$ $67.72, p<.001$, and age, $F(2,14)=29.42$, $p<.0001$. Subsequent comparisons showed that adults made more double-step responses than either group of children, and the 8-10year-olds made more of such responses than the 5-6-year-olds (all $p s<.05$ ). The interaction of intertarget interval and age was not significant, $F(6,42)=2.33, p>.05$.

The finding that the percentage of doublestep responses increased as a function of intertarget interval replicates the experimental results of Wheeless et al. (1966), Komoda et al. (1973), and Carlow et al. (1975) and suggests that it is the extent to which programming has developed at the time of occurrence of the second target that determines whether this response is reprogrammed to the second target. Within this framework the developmental differences in percentage of double-step responses can be most simply interpreted in terms of differences in the rate at which adults and children program saccades. For the children, whose saccadic latency is longer than that of the adult, the

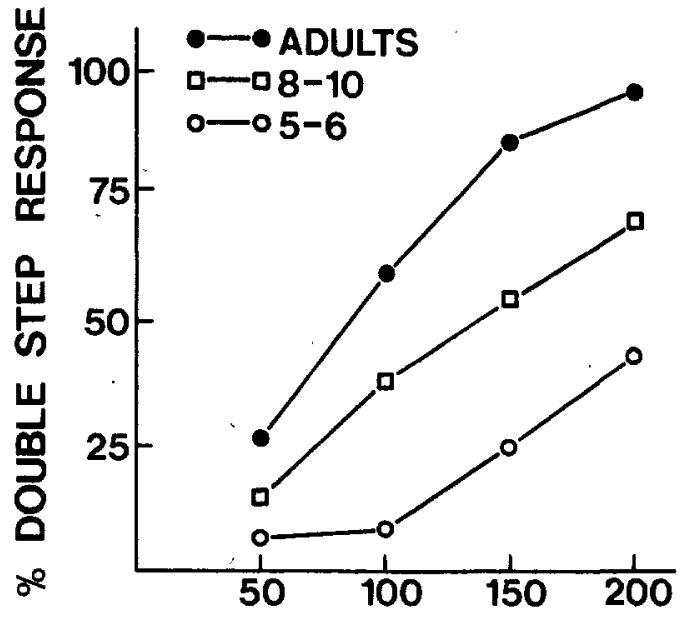

\section{INTERTARGET INTERVAL}

Figure 2. Mean percentage of double-step responses for adults, 8-10-year-olds, and 5-6-year-olds as a function of intertarget interval.

onset of the second target occurs earlier in the programming of the saccade to the first target; and thus the execution of a response to both the first and second targets is less probable. The fact that there was no Age $\times$ Intertarget Interval interaction suggests that there are no qualitative differences in the programming-reprogramming processes of adults and adds further support to a rate of programming interpretation of the age differences in percentage double-step responding.

\section{Saccade Response Latency}

Mean saccade latency was computed for responses to single target displacement, as well as for pulse-over-return and pulse-partial-return trials. In the case of pulse-overreturn and pulse-partial-return trials, either double-step responses to both first and second targets or reprogrammed responses to only the second target could be made. For double-step responses both latencies were computed; the latency of the first response was measured from initial target displacement to the beginning of the first saccade, and the latency of the second response was measured from the onset of the second target to the beginning of the second response. For 
reprogrammed responses the latency of the saccade was measured from the onset of the second target.

Single-step response latency. The primary reason for examining the response latency of single-step responses to single target displacements was to provide a value to which the latencies of reprogrammed responses could be compared, as is discussed in a later section. The effect of extent of target displacement $\left(3^{\circ}\right.$ vs. $\left.6^{\circ}\right)$ on the mean latency of responses to single-step stimuli failed to reach significance for any of the three groups (all $p s>.10$ ), and the data were collapsed across this factor in all subsequent single-step analyses.

Age differences in single-step latency were found, with adults, 8-10-year-olds, and 5-6year-olds responding with mean latencies of 271,330 , and $390 \mathrm{msec}$, respectively. An ANOVA for unequal sample sizes, using unweighted means, found these differences to be significant, $F(2,24)=10.43, p<.01$; and subsequent analyses showed that each age group was significantly $(p<.05)$ different from the others. This finding that adults responded faster than children replicates the results of Cohen and Ross $(1977,1978)$ and is consistent with the latency values that have been reported by previous investigators. The single-step latencies of the 8-10-yearolds and 5-6-year-olds correspond well with the $270 \mathrm{msec}$ reported by Cohen and Ross (1978) and the $410 \mathrm{msec}$ reported by Miller (1969) for comparable age groups. The adults' single-step latency was also well within the range of values reported in the literature (e.g., 284 and $255 \mathrm{msec}$ in the Wheeless et al,, 1966 and Komoda et al., 1973; studies).

Double-step first and second response latencies. To the extent that the programming of successive saccades involves the interaction of separate programs to the disadvantage of the programming of the second response, it might be expected that the degree to which the programming of the first response had been completed at the onset of the second target would be inversely related to the latency of the second response (i.e., the latency of the second response should decrease as the interval between the first and second targets increases). Komoda et al. found this to be true and interpreted the relationship in terms of the longer intertarget interval having the effect of minimizing the time during which ongoing programming of the first response limited the efficiency with which the second response could be programmed. Similar results would be expected in the present data. Age differences in the latencies of both first and second responses were also anticipated, and it was of particular interest to compare children's second response latency intertarget interval relationship to that of adults.

Examination of the first and second saccades in the double-step response indicated that their latencies were not affected by stimulus type at any intertarget interval for any of the age groups (all $p s>.10$ ), and these response latencies were averaged across stimulus type in all analyses.

Table 1 presents the mean latency of the first and second responses for each age group, averaged over stimulus type, as a function of intertarget interval. As can be seen, the effects of intertarget interval on the latency of the first response were minimal; and an ANOVA revealed that the effects were not significant for any of the age groups. Table 1 also shows that there is a trend for the latency of the first saccade to decrease with increasing age; and an ANOVA for unequal sample sizes, conducted on the unweighted means of latencies averaged over intertarget interval, revealed a significant main effect of age, $F(2,14)=7.56, p<.01$. This age effect is again consistent with developmental differences in saccadic latency that have been reported in the literature.

Examination of the second response latency showed decreases as intertarget interval increased for pulse-over-return and pulsepartial-return stimuli for all three age groups. However, since the youngest children had too few double-step responses for analysis at some intertarget intervals, multiple regression/correlation analysis was conducted only on the data of the other two groups. Significant main effects of both age, $F(1,9)=$ $11.97, p<.01$, and intertarget interval, $F(3$, $18)=13.92, p<.001$, were found; but there was no significant interaction of these factors, $F(3,18)=3.36, p>.05$.

The effect of intertarget interval on sec- 
Table 1

Latency of Double-Step Responses

\begin{tabular}{|c|c|c|c|c|c|c|c|c|c|c|}
\hline \multirow{3}{*}{$\begin{array}{l}\text { Age } \\
\text { group }\end{array}$} & \multicolumn{10}{|c|}{ Intertarget interval (msec) } \\
\hline & \multicolumn{2}{|c|}{50} & \multicolumn{2}{|c|}{100} & \multicolumn{2}{|c|}{150} & \multicolumn{2}{|c|}{200} & \multicolumn{2}{|c|}{$M$} \\
\hline & First & Second & First & Second & First & Second & First & Second & First & Second \\
\hline Adults & 252.60 & 427.98 & 248.61 & 400.53 & 252.18 & 361.72 & 266.45 & 362.39 & 254.96 & 388.15 \\
\hline $8-10$ yrs. & 298.00 & 575.33 & 273.68 & 559.01 & 286.52 & 472.72 & 309.32 & 442.07 & 291.88 & 512.28 \\
\hline $5-6$ yrs. & 346.25 & 602.50 & 315.00 & 585.00 & 350.03 & 510.00 & 350.36 & 459.63 & 340.41 & 539.28 \\
\hline$M$ & 298.95 & 535.27 & 279.10 & 514.85 & 296.24 & 448.15 & 308.71 & 421.36 & & \\
\hline
\end{tabular}

ond response latency, which replicates the findings of Komoda et al. (1973), makes intuitive sense. If saccadic programming can occur in parallel to some degree, as demonstrated by Becker and Jürgens (1979), and if parallel programming limits the efficiency with which the second saccade is programmed, as suggested by Komoda et al. (1973), then at longer intertarget intervals the system should have progressed further in processing the first response at the time the second target appears, which could conceivably lead to faster programming of the second response. The failure to find an interaction between second response latency - and intertarget interval indicates that the advantageous effects of longer intertarget intervals are similar for both the adults and 8-10-year-old children.

Reprogrammed response latency. The latency of reprogrammed responses compared to that of responses executed to a single target displacement reflects the additional time or savings in time involved in the cancellation of the initial saccade and the reprogramming of the saccade to the new target. Examination of the latencies of reprogrammed responses to pulse-over-return and pulse-partial-return targets permits a comparison of reprogramming that involves a change in direction versus that which does not. It was expected that the adults' data would replicate the findings of Wheeless et al. (1966) and Komoda et al. (1973) in that the latency of reprogrammed responses on pulse-over-return trials would be longer than that to a single target, which in turn would be longer than reprogrammed response latency on pulse-partial-return trials. That is, the reprogramming of a response to a new target that involved recomputing direction would require full computation time plus the time needed for cancellation of the programming of the first response; whereas reprogramming that involved the same direction but different amplitude could achieve savings from the initial programming and thus show a shorter latency than that of a response to a single target. Age differences in reprogrammed response latency were examined with a primary interest in investigating the possibility that adults and children differed in the extent to which canceling and reprogramming saccadic direction increased response latency or in their ability to re-use previously computed directional information.

Reprogrammed response latencies as a function of stimulus type and intertarget interval are presented in Figure 3, together with response latencies to single-step stimuli, which are shown as horizontal lines. Because several adults did not exhibit reprogrammed responses at intertarget intervals of 200 msec, the mean response latencies of the remaining adults of that interval are presented as unconnected dots. Figure 3 shows that although absolute latencies differ as a function of age, $F(2,14)=10.72, p<.01$, all three age groups show similar latency patterns with respect to stimulus type at the shorter intertarget intervals. In general reprogrammed responses to pulse-over-return stimuli took longer than reprogrammed responses to pulse-partial return stimuli, with single-step response latencies intermediate between these values. Figure 3 also shows that although intertarget interval greatly affected the reprogrammed response latencies of the youngest children, it had only a 


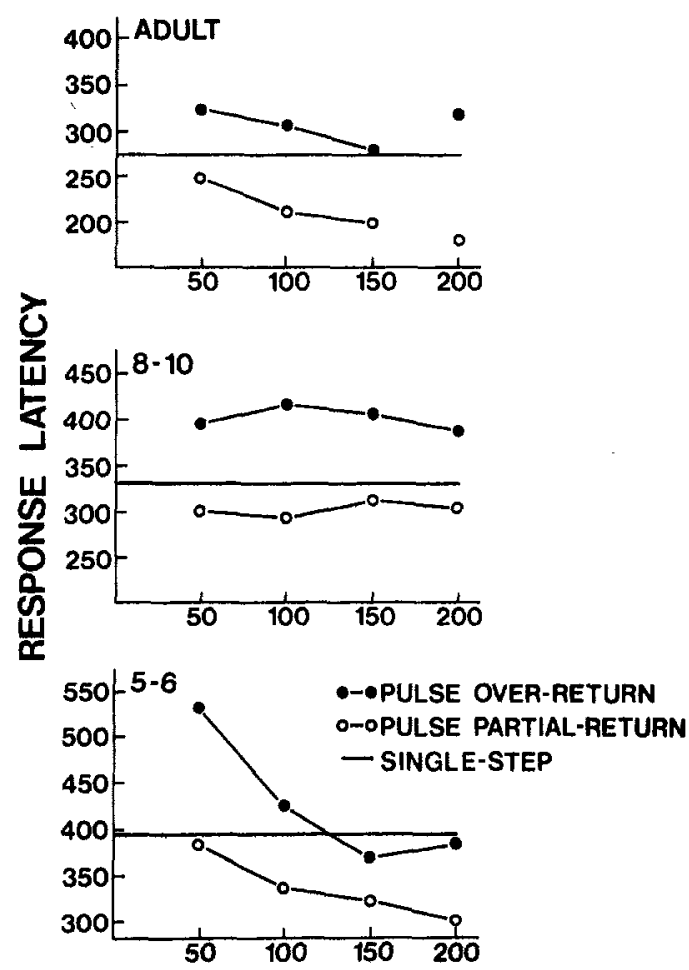

\section{INTERTARGET INTERVAL}

Figure 3. Mean saccadic latency of pulse-over-return and pulse-partial-return reprogrammed responses as a function of intertarget interval for each age group. (The mean single-step response latency for each group is shown as a horizontal line.)

slight effect on the reprogrammed response latencies of adults and virtually no effect at all on the reprogrammed response latencies of the 8-10-year-olds. These findings were supported by ANOVAs for repeated measures designs, which when conducted on the reprogrammed response latencies of each group showed a significant intertarget interval effect only for the 5-6-year-olds, $F(3,12)=$ $6.27, p<.01$.

For the adults and 8-10-year-olds whose latencies were not affected by intertarget interval, multiple regression/correlation analyses with age as a between factor and stimulus type as a within factor were used to separately compare pulse-over-return and pulse-partial-return reprogrammed response latencies, averaged over intertarget interval, to those of the single-step response. The analysis of pulse-over-return data revealed that both age groups took significantly longer to reprogram responses than to execute single-step responses, $F(1,10)=20.18, p<.01$. The adults responded faster than the children, $F(1,10)=8.29, p<.05$; but the interaction of stimulus type and age failed to achieve statistical significance, $F(1,10)=$ $1.2, p>.25$, suggesting that adults and children require similar amounts of additional time to cancel and reprogram responses to pulse-over-return targets. The analysis of the data from pulse-partial-return trials revealed that the latencies of these reprogrammed responses were significantly shorter than those of single-step responses, $F(1$, $10)=37.03, p<.0001$. The age effect was also significant, $F(1,10)=13.41, p<.005$; and in this case the Age $\times$ Stimulus Type interaction was significant, $F(1,10)=10.06$, $p<.01$, indicating that the latency of the adults' reprogrammed responses to pulsepartial-return targets was facilitated to a greater extent than those of children, relative to the latency of the single-step response.

The latency characteristics of the reprogrammed responses and the single-step responses of adults and 8-10-year-old children replicate the findings of previous investigators that latency depends on whether reprogramming involves recomputation of the direction of the saccade. For pulse-over-return reprogrammed responses, in which direction was recomputed, the latency was greater than that of the single-step response, indicating that the saccadic system utilized additional processing time to cancel the first saccade. On the other hand, for pulse-partial-return stimuli, for which first and second targets were displaced in the same direction relative to the fixation point, reprogrammed response latency was facilitated to the point that it was shorter than the latency of the single-step response. It appears that in reprogramming a pulse-partial-return response, the saccadic system is able to utilize the previously computed directional information, thus reducing saccadic latency. The general similarity in the latency relationships of reprogrammed and single-step responses for the adults and children indicates that their saccadic programming and repro- 
gramming mechanisms operate in much the same manner.

Because intertarget interval affected reprogrammed response latency for the 5-6year-old, $t$ tests were conducted between single-step latencies and both pulse-over-return and pulse-partial-return reprogrammed response latencies at each interval. It was found that the pulse-over-return latency was significantly longer only at an intertarget interval of $50 \mathrm{msec}, t(4)=6.14, p<.0$. Reprogrammed responses to pulse-partial-return stimuli were significantly shorter in latency than single-step response latency at intertarget intervals of $100 \mathrm{msec}, t(4)=$ $2.83 ; 150 \mathrm{msec}, t(4)=3.42$; and $200 \mathrm{msec}$, $t(4)=4.25$ (all $p \mathrm{~s}<.05)$.

The youngest children differed from the other age groups in that reprogrammed response latency decreased significantly as intertarget interval increased; in fact at intervals of 150 and $200 \mathrm{msec}$, the 5-6-year-olds responded as quickly as the 8-10-year-olds. It is unclear why such a relationship should be found for this age group.

\section{Amplitude Transition Functions}

Becker and Jürgen's (1979) demonstration that the amplitude of the first saccade to double-step targets changes continuously as a function of the interval from the onset of the second target to the onset of the saccade provides evidence that the saccadic system continuously processes visual information and can modify the amplitude of a saccade in preparation. The finding of amplitude transition functions for both the children and adults of the present study would provide additional evidence that the programming characteristics of these groups are qualitatively similar. Accordingly, the amplitude data from pulse-partial-return and pulse-over-return trials were plotted for each subject as a function of the delay from the onset of the second-target to the occurrence of the first response, or " $D$ " in Becker and Jürgens' terminology. Since the warning interval variable introduced in Experiment 2 did not appear to affect amplitude transition functions, the following comments apply to the data from both experiments.
Becker and Jürgens reported a continuous amplitude transition function from the initial target position to the second target position on pulse-partial-return trials as $D$ increased. However, in the case of pulse-overreturn trials, where a reprogramming of direction is required, a discontinuity in response amplitude was found in that amplitude transition functions extended for a short segment from the first target saccade location but terminated prior to the fixationpoint abscissa value.

Following Becker and Jürgens, examples of amplitude transition functions are shown in Figure 4, with the horizontal dashed lines corresponding to the individual subject's average response amplitude to single steps of $3^{\circ}$ or $6^{\circ}$. Also as in Becker and Jürgens, an oblique amplitude transition function was fitted by eye to the pulse-partial-return trial data. An attempt was made to compute Becker and Jürgens's modification time measure (i.e., the minimum delay for the second target to modify the amplitude of the saccade prepared in response to the first target), but the variability of the data precluded reliable estimates for too many subjects to make the comparisons meaningful.

Interpretation of the amplitude transition functions plotted for the subjects of the present study presented some difficulties since the practical constraints of working with young children limited the number of trials that could be given and thus the number of data points available for analysis. Also the data were quite variable, which may at least partly reffect the fact that these subjects, unlike those in the Becker and Jürgens's study, were naive with respect to serving in visual experiments and showed significant age differences in number of double-step responses. Despite these limitations examination of the data revealed that the great majority of subjects in all age groups (19 of the 23 adults and 21 of the 27 children in Experiments 1 and 2 ), could be identified as exhibiting evidence of amplitude transition functions on pulse-partial-return trials and separate amplitude branches on pulse-overreturn trials. These results replicate those of Becker and Juirgens except for the fact that only one subject, an adult, demonstrated a 
Pulse-Partial-Return

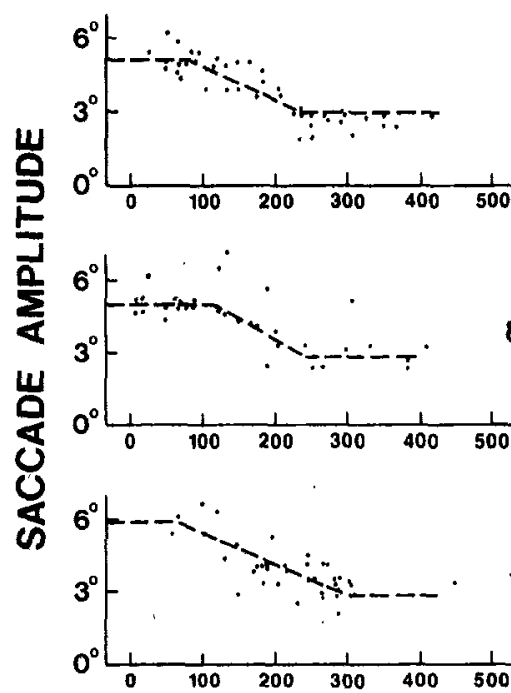

\section{Pulse-Over-Return}

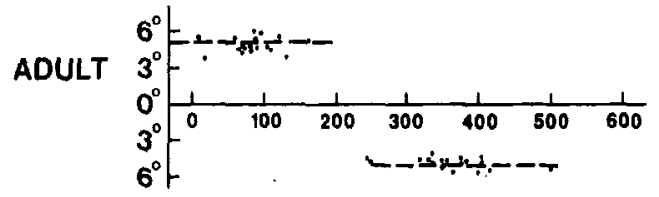

CHILD

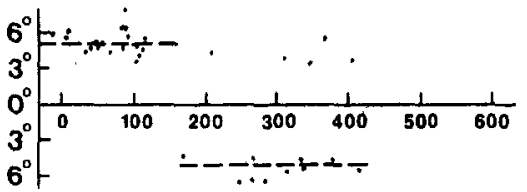

CHILD

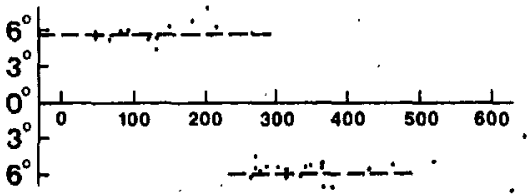

\section{$D(\mathrm{msec})$}

Figure 4. Examples of amplitude transition functions for pulse-partial-return and pulse-over-return trials. (The fixation point is $0^{\circ}$ and the amplitude of the first saccade is shown as a function of the interval from the onset of the second target to the onset of the first response. Horizontal lines correspond to the subject's average response amplitude to single steps of $3^{\circ}$ or $6^{\circ}$.)

pulse-over-return trial amplitude transition segment that extended from the first target position. The lack of such segments might be expected, however, under some reaction time and intertarget interval conditions.

The general similarity of the amplitude transition function data for children and adults suggests that these groups did not differ greatly in terms of their ability to continuously process visual information and use new information to modify the response amplitude of the saccade being programmed.

\section{Intersaccadic Interval and Parallel Programming}

The second aspect of Becker and Jürgens's analysis of double-step responding that is of particular interest for the present study concerns the relationship of the double response intersaccadic interval to the delay interval, D. Since the D interval represents the time available for the parallel processing of first and second targets, a negative slope to the relationship between the intersaccadic inter- val and $\mathrm{D}$ is taken as a demonstration that some computational steps for the second response must have been completed simultaneously with the preparation of the first saccade (see Becker \& Jürgens, 1979, for a discussion of this point). In order to determine if such a negative relationship existed in the present data, scatterplots of the intersaccadic interval - D relationship were examined; and a standardized regression coefficient was computed for each subject. The mean regression coefficient values for pulse-over-return and pulse-partial-return responses were, respectively, -.283 and -.338 for adults, .115 and -.010 for $8-10$ year-olds, and -.149 and -.100 for 5-6year-olds. An ANOva of these data failed to find a significant age effect in the case of either response. Examination of the individual subject data revealed that four adults had negative values on pulse-over-return trials and all five had negative values on pulse-partial-return trials, with the corresponding numbers on the respective trial types two and two for 8-10-year-olds and 
four and three for 5-6-year-olds. Although the small number of subjects in the groups and the fact that children made fewer double step responses than adults make any conclusions very tentative, there is a slight suggestion in these data of a more consistently negative relationship between the intersaccadic interval and D on the part of adults. Because a slope of this relationship with a value between 0 and -1 presumably indicates that some parts of the concurrent preparation of the second saccade were carried out at reduced speed (see Becker \& Jürgens, 1979), less negative slopes on the part of children's functions could indicate slower simultaneous programming of the second response on the part of some of these subjects. A further analysis of the intersaccadic interval - D relationship was carried out with a larger number of adults and 8-10-year-old children in Experiment 2, as reported below.

\section{Experiment 2}

Warning signals have been shown to improve subject's reaction times in a variety of tasks, with the great majority of studies involving manual reaction time to visual or auditory stimuli. Although the effects of warning signals on saccadic eye movements have been much less extensively studied, a number of experiments have demonstrated that a warning signal reduces saccade latency to a visual target. Saslow (1967), who used fixation-point offset as a warning event, found an optimum facilitating effect on saccadic latency when the warning signal occurred approximately $300 \mathrm{msec}$ prior to target onset. Becker (1972) reported that fixation point offset only $120-160 \mathrm{msec}$ prior to target onset reduced saccadic latency by $80 \mathrm{msec}$, even though the blackout period was not reported by the subjects as perceptible.

In a later study, Cohen and Ross (1978) replicated the warning effect results of Becker (1972) and Saslow (1967) and also Miller's (1969) finding that under conditions of no warning, adults' saccades were faster than children's. Cohen and Ross also found, however, that although the children's saccades were slower than those of the adults under no warning or 100 -msec warning-in- terval conditions, the children's responses were as fast as those of adults with longer warning intervals of 300 or $600 \mathrm{msec}$. Cohen and Ross concluded that age differences in saccadic latency were due to functional differences in the amount of time needed by adults and children to process information, rather than fixed central processing limitations on the part of children. A later study (Cohen \& Ross, 1978) replicated these findings and demonstrated that the greater improvement shown by children with longer warning intervals was not the result of a speed-accuracy trade-off.

Given the experimental finding that the occurrence of a warning event can facilitate saccadic responses, it was of interest to determine the effects of warning on the saccades of both children and adults to doublestep targets. In particular it might be expected that with warning the saccadic system would be at a later processing stage at the onset of the target, which could influence further programming of saccades, perhaps differently for children and adults. Also, it was unknown whether the facilitating effects of a warning signal that is noninformative with respect to the direction or extent of the target would affect subsequent saccades, such as the second saccade in a double-step response, or a saccade that involved reprogramming. In order to provide this information, Experiment 2 investigated the effects of three warning intervals- $-0,100$, and $300 \mathrm{msec}$-on the saccadic responses of children and adults to double-step stimuli.

\section{Method}

\section{Subjects}

Subjects consisted of 18 college students and 15 children who were recruited through friends and acquaintances. The adults were randomly assigned to three groups: no warning, 100-msec warning, or 300-msec warning, with four women and two men in each group. The mean ages of the children were 8.7 years $(S D=$ $.72)$ and 8.7 years $(S D=.74)$ in the no-warning and $100-\mathrm{msec}$ warning groups, respectively. The mean age of the children in the $300-\mathrm{msec}$ group, was 9.9 years $(S D=.99)$. There were three girls and two boys in each group, with each child either given a toy or paid $\$ 2$ for his or her time. All subjects were required to have at least 20/30 vision, without corrective lenses, as measured by the Titmus tester. 


\section{Apparatus, Stimuli, and Procedure}

The apparatus and stimuli were identical to that of Experiment 1, as were the experimental conditions and the procedure for the no-warning group. The fixation point was extinguished $100 \mathrm{msec}$ and $300 \mathrm{msec}$ prior to target onset for the 100 and $300 \mathrm{msec}$ warning conditions.

\section{Results and Discussion}

Preliminary analysis of left-right differences again revealed no significant effect of initial direction, and all response measures were averaged over these conditions.

\section{Percentage of Double-Step Responses}

Experiment 1 revealed that the percentage of double-step responses increased with increasing intertarget interval for both adults and children, with adults executing a significantly greater number of double-step responses than the younger groups. Similar results were expected in the warning groups in Experiment 2, with the question of interest being whether the presence of a 100- or 300msec warning interval would advance the programming of the first response and thus increase the probability of its execution.

The percentage of double-step responses for each age group and warning-interval combination is shown in Figure 5. As in Experiment 1, there was no significant effect of stimulus type on percentage of double-

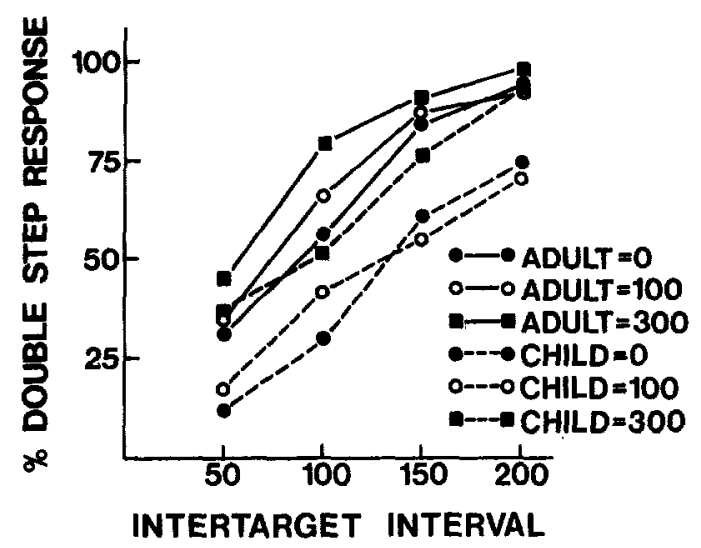

Figure 5. Mean percentages of double-step responses as a function of intertarget interval for adults and children under conditions of no warning, $100-\mathrm{msec}$ warning and 300-msec warning. step responses for any of the six groups, $F(1$, $4)=2.13, p>.10$, for the 100 -msec warning-interval children's group; and $F<1$ for all other groups. Multiple regression/correlation analysis with age and warning as between factors and intertarget interval as a within factor revealed a significant main effect of intertarget interval, $F(3,93)=$ $147.09, p<.0001$. These results are similar to those of the first experiment, as in the finding that adults executed a significantly greater number of double-step responses than did the children, $F(1,31)=12.3$, $p<.025$.

Figure 5 also shows that the subjects under the warning conditions generally executed a greater number of double-step responses, particularly at the shorter intertarget intervals; however, the warning factor was not significant, $F(2,29)=1.89, p>.10$. Although it appears that with a 300 -msec warning interval, the children's double-step response level increased to a greater degree than that of the adults; the interaction of Age $\times$ Warning Interval was not significant nor were any of the interactions involving other factors (all $F \mathrm{~s}<1$ ). It should be noted that with a $300-\mathrm{msec}$ warning interval, the children's frequency of double-step response was comparable to that of adults with no warning.

\section{Saccade Response Latency}

Because a warning interval has been demonstrated to reduce saccade latency in both adults and children, similar warning effects were anticipated for single-step responses and for the first saccade of double-step responses. However, it was questionable whether the effects of nonspecific warning intervals such as the $100-$ or $300-\mathrm{msec}$ periods used in Experiment 2 would affect responses that were programmed in succession (i.e., the second saccade in the double-step response) and single saccades to the second target, which involved reprogramming. It was of particular interest to investigate the possibility that the presence of a warning interval affected the way in which directional information was reprogrammed or reused in the reprogrammed responses and 
that this might occur differently for children and adults.

Single-step response latency. As in the first experiment, ANOVAs revealed that extent of target displacement $\left(3^{\circ}\right.$ vs. $\left.6^{\circ}\right)$ did not affect response latency significantly $(p>$ .25 for all groups), and the data were averaged across displacement extent in all subsequent analyses involving responses to single-step stimuli.

There were age differences in response latency to single-step targets as in Experiment 1 and also a warning effect for both age groups. Mean latencies for conditions of no warning, 100-msec warning, and 300msec warning, respectively, were 255,241 , and 205 msec for adults; and 330, 300, and $244 \mathrm{msec}$ for children. A $2 \times 3$ ANOVA for unequal sample sizes performed on the unweighted means of single-step latencies of children and adults with age and warning as between factors revealed a main effect of both age, $F(1,27)=10.71, p<.01$, and warning, $F(2,27)=5.03, p<.025$. The Age $\times$ Warning interaction was not significant $(F<1)$.

The single-step response latencies for adults and children in the no-warning conditions were consistent with the latencies of the comparable groups in the first experiment, and the warning effects for adults replicated the findings of Saslow (1967) and Becker (1972). Warning facilitated the children's saccadic latency to a lesser extent than was reported by Cohen and Ross (1977), with adults still responding with shorter latencies than children who had a 300-msec warning interval.

Double-step first and second response latency. Examination of the latency of responses to the first target on trial where double-step responses were executed revealed no systematic differences between responses to pulse-over-return and pulse-partial-return stimuli (all $p s>.25$ ), and first response latencies were therefore collapsed across stimulus type for subsequent analyses.

The mean latencies of the first response for 0-, 100-, and 300-msec warning intervals were respectively 293,256 , and $215 \mathrm{msec}$ for children and 241, 221, and $198 \mathrm{msec}$ for adults. A $2 \times 3$ unweighted mean ANOVA for unequal sample sizes with age and warning as between factors revealed a main effect of both age, $F(1,27)=22.63, p<.0001$, and warning, $F(2,27)=22.32, p<.001$, but no interaction of these factors, $F(2,27)=1.99$, $p>.10$. The single-step data from the present experiment also showed adults responding faster than children at warning intervals of $300 \mathrm{msec}$.

Again as in the first experiment, the latency of the second response tended to decrease as intertarget interval increased, in this case for all six groups. ANOVAs performed on these data with intertarget interval and stimulus type as within factors revealed a main effect of intertarget interval for adults under all three warning conditions: $F(2,10)=17.93,16.3$, and 19.9 , all $p s<.001$, for warning intervals of 0 -, $100-$, and $300-\mathrm{msec}$, respectively; and for children under the 100- and 300-msec warning conditions, $F(2,8)=200.44$ and 9.4 , $p s<.01$. Several children under the nowarning condition did not exhibit doublestep responses at intertarget intervals of 50 and $100 \mathrm{msec}$, and no analysis was performed on the data of this group. As was the case of the second response latencies in Experiment 1 , neither the effects of stimulus type nor the interaction of stimulus type and intertarget interval were significant for any of the five groups analyzed.

With respect to the effects of warning on the latency of the second response, an ANova performed on both the adults' pulseover-return and pulse-partial-return data, with warning as a between factor and intertarget interval as a within factor, revealed that there was no effect of warning for either pulse-over-return trials or pulse-partial-return trials, $F(2,15)=1.08$ and $1.0, p s<$ .25. Furthermore, none of the interactions involving these factors was significant (all $F \mathrm{~s}<1$ ). Thus it appears that although warning facilitates the first of the saccades in a double-step response, its effects do not carry over to the subsequent response. It is possible that nonspecific warning effects are effectively reset to zero when a new target initiates programming or that the programming that occurs to a stimulus event in itself serves as warning for subsequent responses so that additional extraneous warning is redundant and has no effect. With respect to 
the latter possibility, Becker (1972) has suggested that a main saccade could act as a warning event for corrective saccades, thus reducing their latency.

Reprogrammed response latency. $\mathrm{Re}-$ programmed response latencies for the six groups are presented as a function of stimulus type and intertarget interval in Figure 6 , together with the latency values for responses to single-step targets that appear on the figure as unbroken lines. Intertarget interval appears to have affected reprogrammed response latencies in some cases, but an ANOVA for repeated measures designs for each group with stimulus type and intertarget interval as within factors revealed that the effect of intertarget interval was significant only for the adults under the nowarning condition, $F(2,10)=9.22, p<.01$. Intertarget interval did not significantly affect the reprogramming time of any other group, nor were the stimulus type by intertarget interval interactions significant (all ps > .05).

Figure 6 shows that the adults and children in the no-warning groups exhibited similar latency patterns to those of the adults and 8-10-year-olds of Experiment 1 in that single-step responses were executed more quickly than reprogrammed responses to pulse-over-return targets, and reprogrammed responses to pulse-partial-return

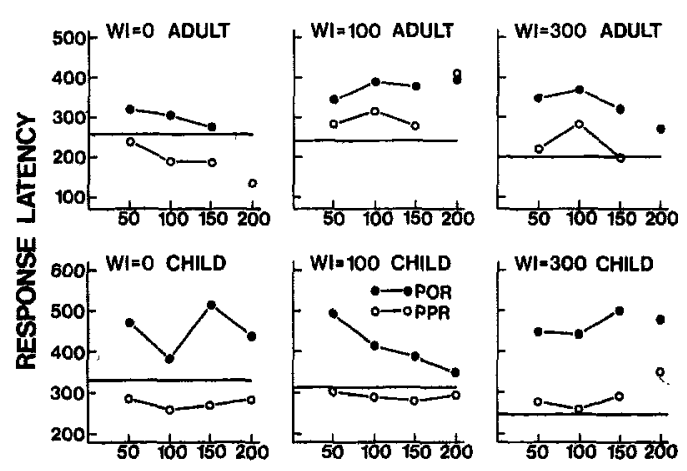

INTERTARGET INTERVAL

Figure 6. Mean saccadic latencỳ of pulse-over-return and pulse-partial-return reprogrammed responses as a function of intertarget interval for adults and children under conditions of no warning, $100 \mathrm{msec}$ warning, and $30 \mathrm{msec}$ warning. (Single step latencies are shown as a horizontal line for each group.) targets were executed more quickly than either. As can be seen, the presence of a warning interval greatly changed the relationships between the various reprogrammed response latencies and that of the single-step response for both adults and children. However, examination of the pattern changes in latency that occur with warning reveals that they result primarily from decreases in the latency of the single-step responses. Warning had little if any effect on reprogrammed response latencies with pulse-overreturn and pulse-partial-return single-step responses showing generally similar latency values under 0-, 100-, and 300-msec warning. Subsequent analyses of the effects of warning and age on reprogrammed response latency revealed that there was no effect of warning for either pulse-over-return trials $(F<1)$ or pulse-partial-return trials, $F(2$, $27)=1.68, p>.10$. Thus as was the case for the second saccade in the double-step response, warning did not affect reprogrammed response latency, and the same interpretation appears to apply (i.e., that the initial warning had no effect because the original programming served as warning for the subsequent modifications or that the warning effect was reset to zero when the saccadic programming of the first response was initiated). Although adults tended to reprogram responses faster than children, the effect of age failed to achieve significance for either pulse-over-return trials $(F<$ 1) or pulse-partial-return trials, $F(1,27)=$ $2.35, p>.10$. The Age $\times$ Warning interactions were also nonsignificant (all $F \mathrm{~s}<1$ ).

\section{Intersaccadic Interval and Parallel Programming}

As in Experiment 1, scatterplots of the relationship between the intersaccadic interval and the delay (D) from second stimulus onset to the first response were examined and standardized regression coefficients computed for each subject. Table 2 presents the mean regression value for each group for pulse-over-return and pulse-partial-return trials. Although the overall slope values again were less negative for children than for adults, there were reversals in that the children's mean slope was more negative 
Table 2

Mean Standardized Regression Coefficients Showing Intersaccadic Interval as a Function of Delay (D) From Second Target Onset to First Saccade

\begin{tabular}{lccccc}
\hline & \multicolumn{2}{c}{ Adults } & & \multicolumn{2}{c}{ Children } \\
\cline { 2 - 3 } $\begin{array}{c}\text { Warning interval } \\
\text { condition }\end{array}$ & $\begin{array}{c}\text { Pulse-over- } \\
\text { return }\end{array}$ & $\begin{array}{c}\text { Pulse-partial- } \\
\text { return }\end{array}$ & & $\begin{array}{c}\text { Pulse-over- } \\
\text { return }\end{array}$ & $\begin{array}{c}\text { Pulse-partial- } \\
\text { return }\end{array}$ \\
\hline No warning & $-.477(4 / 5)$ & $-.279(4 / 5)$ & & $-.323(4 / 5)$ & $-.331(4 / 5)$ \\
$100-$-msec warning & $-.254(6 / 6)$ & $-.086(4 / 6)$ & & $-.033(2 / 5)$ & $.233(1 / 5)$ \\
300 -msec warning & $-.502(6 / 6)$ & $-.194(4 / 6)$ & & $-.228(3 / 5)$ & $-.265(5 / 5)$ \\
\hline
\end{tabular}

Note. The number of subjects who had negative slope values and the total number of subjects in the group are shown in parentheses.

than adults on pulse-partial-return trials under both 0 - and 300-msec warning-interval conditions. An ANOVA of the regression coefficient data with age, trial type, and warning interval as the factors found only warning interval to be significant, $F(2,26)=3.75$. Subsequent $t$ tests comparing the warning interval conditions found the slope to be less negative with $100-\mathrm{msec}$ warning than with 0 - or $300-\mathrm{msec}$ warning (both $p \mathrm{~s}<.01$ ), whereas the $0-$ and 300 -msec warning conditions did not differ significantly. There are some reasons to expect more negative slope values for pulse-over-return than pulse-partial-return trials, but the trial type variable was not significant nor was the age variable in a separate analysis of the pulse-over-return trial data.

Thus although there are some further indications of a less negative relationship between intertrial interval and D on the part of children, the differences were not statistically significant, and the data are inconclusive with respect to this point. These results do suggest that a further examination of age differences in the intersaccadic interval - D relationship could be fruitful. Extended sessions would be necessary, however, to provide sufficient data from individual subjects to permit a clear answer to the question of age differences in the parallel processing of saccades.

The finding of a significant effect of warning interval on the slope of the intersaccadic interval - D function is interesting in that it could imply some incompatibility between steps of the programming sequence and the efficiency of parallel programming, depend- ing upon the manner in which nonspecific warning affects particular steps of the programming sequence. Because there is little information about such effects and their time course, at the present time, it is not clear just what mechanism might underlie this warning interval - slope relationship.

\section{General Conclusions}

The results of Experiments 1 and 2 demonstrate that the saccadic responses of adults and children to double-step stimuli differ primarily in percentage of double-step responses and saccadic latency. Strong similarities in response patterns were found under most conditions, with all age groups demonstrating increases in frequency of double-step responding with increases in intertarget interval, longer latencies for reprogrammed responses that involved recomputing direction as compared to those that did not, and modifiability of saccadic programming (i.e., amplitude transition functions). Both adults and children also demonstrated parallel processing of saccades, although the data suggested the possibility of age-related differences in the speed of simultaneous saccadic programming. For both groups the occurrence of a warning event was accompanied by increases in percentages of double-step responding, as well as decreases in the latencies of both the first saccade in the double-step response and the single response to single target displacement, with no effect on the latencies of either the second saccade in the double-step response or reprogrammed responses. In ad- 
dition warning affected the slope of the intersaccadic interval D function (i.e., the efficiency with which saccades were programmed in parallel for both adults and children). However, the nature of the warning interval - slope relationship is unclear. Thus the data support the position that age differences in percentages of double-step responding and saccadic latency are a function of differences in the rate at which adults and children program and execute saccades, rather than qualitative differences in saccadic programming mechanisms.

The similarities in characteristics of double-step responding of adults and children in these two experiments suggest that developmental changes in saccadic programming occur primarily with respect to the rate with which the saccade is programmed. However, the relationship between age changes in saccadic latency and developmental differences in higher level visual activities such as scanning is probably quite complex. It is possible that age changes in the eye movement control system, such as decreases in saccadic latency, are accompanied by more efficient intake of visual information during complex visual activities. Indeed it has been suggested that eye movements themselves play a role in the development of schemata (Jeffrey, 1968) and also that age changes in complex patterns of eye movements are related to the emergence of efficient scanning strategies in visual search tasks (Day, 1975). To the extent that saccadic latency is related to visual performance across a wide variety of tasks, one might expect the age differences in the latency of saccadic programming exhibited in double-step responding to contribute to developmental changes in more complex visual and cognitive activities.

\section{References}

Aslin, R. N., \& Salapatek, P. Saccadic localization of visual targets by the very young human infant. Perception \& Psychophysics, 1975, 17, 293-302.

Becker, W. The control of eye movements in the saccadic system. In J. Dichgans \& E. Bizzi (Eds.), Ce- rebral control of eye movements and motion perception. Basel, Switzerland: Karger, 1972.

Becker, W., \& Fuchs, A. F. Further properties of the human saccadic system: Eye movements and corrective saccades with and without visual fixation points. Vision Research, 1969, 9, 1247-1258.

Becker, W., \& Jürgens, R. An analysis of the saccadic system by means of double-step stimuli. Vision Research, 1979, 19, 967-983.

Carlow, T., Dell-Osso, L. F., Troose, B. T., Daroff, R. B., \& Birkett, J. E. Saccadic eye movement latencies to multimodal stimuli. Vision Research, 1975, 15, 1257-1262.

Cohen, M. E., \& Ross, L. E. Saccade latency in children and adults: Effects of warning interval and target eccentricity. Journal of Experimental Child Psychology, 1977, 23, 539-549.

Cohen, M. E., \& Ross, L. E. Latency and accuracy characteristics of saccades in children and adults. Journal of Experimental Child Psychology, 1978, 26, 517-527.

Day, M. C. Developmental trends in visual scanning. In $\mathrm{H}$. Reese (Ed.), Advances in child development and behavior (Vol. 10). New York: Academic Press, 1975.

Fellows, B. J. Chance stimulus sequences for discrimination tasks. Psychological Bulletin, 1967, 67, 8792.

Jeffrey, W. E. The orienting reflex and attention in cognitive development. Psychological Review, 1968, 75, 323-334.

Komoda, M. K., Festinger, L., Phillips, L. J., Duckman, R. H., \& Young, R. A. Some observations concerning saccadic eye movements. Vision Research, 1973, 13, 1009-1020.

Miller, L. K. Eye-movement latency as a function of age, stimulus uncertainty, and position in the visual field. Perceptual and Motor Skills, 1969, 28, 631636.

Saslow, M. G. Effects of components of displacementstep stimuli upon latency for saccadic eye movement. Journal of the Optical Society of America, 1967, 57, 1024-1029.

Taumer, $R$. Three reaction mechanisms of the saccadic system in response to a double jump. In $G$. Lennerstrand \& P. Bach-y-Rita (Eds.) Basic mechanisms of ocular motility. Oxford, England: Pergamon Press, 1975.

Vurpillot, E. The development of scanning strategies and their relation to visual differentiation. Journal of Experimental Child Psychology, 1968, 6, 632-650.

Wheeless, L. L., Jr., Boynton, R. M., \& Cohen, G. H. Eye-movement responses to step and pulse-step stimuli. Journal of the Optical Society of America, 1966, 56, 956-960.

Whiteside, J. A. Eye movements of children, adults, and elderly persons during inspection of dot patterns. Journal of Experimental Psychology, 1974, 18, 313332 .

Received August 4, 1981 